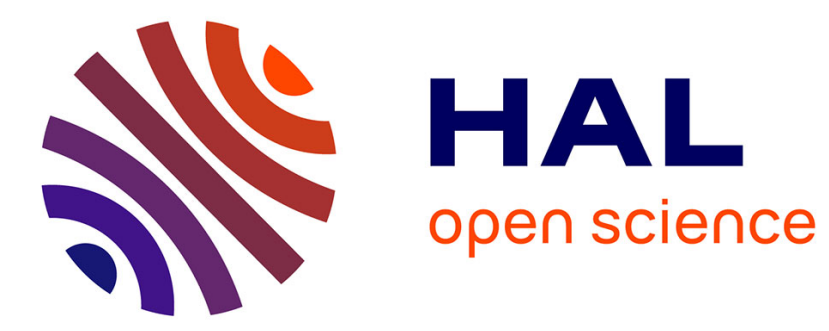

\title{
Charge distribution and kinetic pressure in a plasma : a soluble model
}

\author{
B. Jancovici
}

\section{To cite this version:}

B. Jancovici. Charge distribution and kinetic pressure in a plasma: a soluble model. Journal de Physique Lettres, 1981, 42 (11), pp.223-226. 10.1051/jphyslet:019810042011022300 . jpa-00231914

\section{HAL Id: jpa-00231914 https://hal.science/jpa-00231914}

Submitted on 1 Jan 1981

HAL is a multi-disciplinary open access archive for the deposit and dissemination of scientific research documents, whether they are published or not. The documents may come from teaching and research institutions in France or abroad, or from public or private research centers.
L'archive ouverte pluridisciplinaire HAL, est destinée au dépôt et à la diffusion de documents scientifiques de niveau recherche, publiés ou non, émanant des établissements d'enseignement et de recherche français ou étrangers, des laboratoires publics ou privés. 


\title{
LE JOURNAL DE PHYSIQUE-LETTRES
}

\section{Charge distribution and kinetic pressure in a plasma : a soluble model}

\author{
B. Jancovici \\ Laboratoire de Physique Théorique et Hautes Energies, Université Paris-Sud, 91405 Orsay, France \\ (Reçu le 9 mars 1981, accepté le 3 avril 1981)
}

\begin{abstract}
Résumé. - Pour un plasma classique à une composante en deux dimensions (avec une interaction logarithmique), on peut calculer exactement la densité à un corps quand la constante de couplage a la valeur particulière $\Gamma=2$. On étudie le comportement de cette densité près de la paroi de la boîte circulaire qui contient le système. S'il y a une charge en excès, elle se concentre près de la paroi. La densité sur la paroi est reliée à la pression cinétique, qui diffère de la pression thermique.
\end{abstract}

\begin{abstract}
For a classical two-dimensional one-component plasma (with a logarithmic interaction), the onebody density can be calculated exactly when the coupling constant has the special value $\Gamma=2$. The behaviour of this density is studied near the wall of the circular box which encloses the system. If there is an excess charge, it concentrates near the wall. The density at the wall is related to the kinetic pressure, which differs from the thermal pressure.
\end{abstract}

1. Introduction. - Recently, exact results have been obtained on the equilibrium statistical mechanics of a classical two-dimensional one-component plasma [1], through methods of the theory of random matrices [2]. The model is a system of $N$ identical particles of charge $e$ embedded in a uniform neutralizing background of opposite charge. In two dimensions, the Coulomb interaction potential between two particles at a distance $r$ from one another is

$$
v(r)=-e^{2} \ln (r / L),
$$

where $L$ is a length scale. The dimensionless coupling parameter is $\Gamma=e^{2} / k_{\mathrm{B}} T$, where $k_{\mathrm{B}}$ is Boltzmann's constant and $T$ is the temperature. At the special value $\Gamma=2$, it is possible to compute exactly the thermodynamic functions and the distribution functions. In the present Letter, we study in some detail the one-body distribution function for a system of particles confined in a disc, with special attention to the behaviour of this function near the edge of the disc.

The one-body distribution is interesting in itself; here is a model of interacting charged particles for which it is possible to compute without approxima- tions how a wall distorts the distribution in its neighbourhood. A non-neutral system can also be treated, and it is amusing to be able to see how the excess charge concentrates near the walls as expected.

The model also provides an illustration for the different non-equivalent " pressures " in a one-component plasma [3, 4]. If a «thermal pressure» $p^{(\theta)}$ is defined in the usual way by differentiating the free energy with respect to the volume, keeping constant the total charge of the particle plus background system, the resulting equation of state has the simple exact form [5]

$$
p^{(\theta)}=\left(k_{\mathrm{B}} T-\frac{1}{4} e^{2}\right) \rho,
$$

where $\rho$ is the number density. This thermal pressure becomes negative at low temperatures (the same phenomenon also occurs in three dimensions). A negative pressure for a one-component plasma is not a serious trouble, since the background can be considered as maintained rigid at a given density by some external constraint. However, it is possible to give other definitions of the pressure, not all of which are equivalent in the case of a one-component 
system with long-range forces. One of these definitions is the "kinetic pressure " which is exerted by the particles on the walls; for a spherical box, this pressure is

$$
p^{(\mathbf{k})}=\rho_{\mathrm{W}} k_{\mathrm{B}} T,
$$

where $\rho_{\mathrm{w}}$ is the particle density at the wall. This kinetic pressure is always positive. It can also be obtained by a proper use of the virial theorem. In the present model, at $\Gamma=2$, it is possible to compute the exact value of $\rho_{\mathrm{w}}$ and therefore of $p^{(\mathbf{k})}$, and to verify that a general expression [4] for $p^{(\mathbf{k})}-p^{(\boldsymbol{\theta})}$ is indeed valid.

2. Neutral system. - We consider a system of $N$ particles of charge $e$ in a disc of radius $R$. The disc is filled with a uniform background of total charge - Ne. An average interparticle distance is defined as $a=R / \sqrt{N}$. The average particle number density is $\rho=N / \pi R^{2}=1 / \pi a^{2}$. The distance $r$ to the disc centre can be expressed by the dimensionless $\left(^{1}\right)$ variable $z=r / a$. When $\Gamma=2$, using the method described in reference [1], one finds [2] for the onebody density $\rho(z)$

$$
\frac{\rho(z)}{\rho}=\mathrm{e}^{-z^{2}} \sum_{n=0}^{N-1} \frac{z^{2 n}}{\gamma(n+1, N)},
$$

where

$$
\gamma(n+1, N)=\int_{0}^{N} \mathrm{e}^{-t} t^{n} \mathrm{~d} t
$$

is the incomplete gamma function.

If we take the thermodynamic limit $N \rightarrow \infty$ for fixed values of $\rho$ and of the distance $z, \rho(z)$ goes to the constant value $\rho$ : away from the walls, the particle charge density is a constant which is exactly compensated by the background charge density. We are more interested however in the behaviour of $\rho(z)$ in the disc at some fixed distance from the edge. The edge is at $z=\sqrt{N}$. Therefore, we set $z=\sqrt{N}-y$, and look for the limit of (2.1) as $N \rightarrow \infty$, for fixed values of $\rho$ and $y$. This limit is computed as follows. We assume that the important values of $n$ in (2.1) are such that $N-n$ is of the order of $\sqrt{N}$ (this will be verified a posteriori). An asymptotic expression for $\gamma(n+1, N)$ can then be obtained by rewriting the integrand in (2.2) as $\exp (-t+n \ln t)$, expanding $-t+n \ln t$ around its maximum at $t=n$ up to the order $(t-n)^{2}$, and extending the integration range of $t$ to $(-\infty, N)$. One finds [6]

$$
\begin{array}{r}
\gamma(n+1, N)=(2 \pi n)^{1 / 2} \exp (-n+n \ln n) \times \\
\times \frac{1}{2}\left[1+\Phi\left(\frac{N-n}{\sqrt{2 n}}\right)+\mathrm{O}\left(\frac{1}{\sqrt{n}}\right)\right],
\end{array}
$$

( ${ }^{1}$ In reference [1], $z$ was defined with a different normalization. where

$$
\Phi(x)=\frac{2}{\sqrt{\pi}} \int_{0}^{x} \exp \left(-t^{2}\right) \mathrm{d} t
$$

is the error function. (If $(N-n) / \sqrt{2 n} \gg 1,(2.3)$ is just Stirling's formula.) Using (2.3) in (2.1), we obtain

$$
\begin{aligned}
\frac{\rho(z)}{\rho}=\sum_{n=0}^{N-1} \frac{2 \exp \left(-z^{2}+n \ln z^{2}+n-n \ln n\right)}{(2 \pi n)^{1 / 2}} & {\left[1+\Phi\left(\frac{N-n}{\sqrt{2 n}}\right)\right] } \\
\times & {\left[1+\mathrm{O}\left(\frac{1}{\sqrt{N}}\right)\right] . }
\end{aligned}
$$

Expanding the argument of the exponential in (2.4) as a function of $n$ around its maximum at $n=z^{2}$ up to the order $\left(n-z^{2}\right)^{2}$, discarding terms of order $1 / \sqrt{N}$, and using the variable $x=(N-n) / \sqrt{2 N}$, in the thermodynamic limit one transforms the sum (2.4) into the integral

$$
\frac{\rho(y)}{\rho}=\frac{2}{\sqrt{\pi}} \int_{0}^{\infty} \frac{\exp \left[-(x-y \sqrt{2})^{2}\right]}{1+\Phi(x)} \mathrm{d} x,
$$

where the position is now defined by $y$, the distance to the edge in units of $a$. Since the integral (2.5) converges as $x \rightarrow \infty$, our assumption that the relevant values of $N-n$ are of the order of $\sqrt{N}$ is justified.

For $y=0$, i.e. at the wall on the disc edge, one finds from (2.5)

$$
\rho_{\mathrm{W}}=\rho(0)=\rho \ln 2,
$$

and the corresponding kinetic pressure is given by (1.3).

For large values of $y$, i.e. far away from the walls,

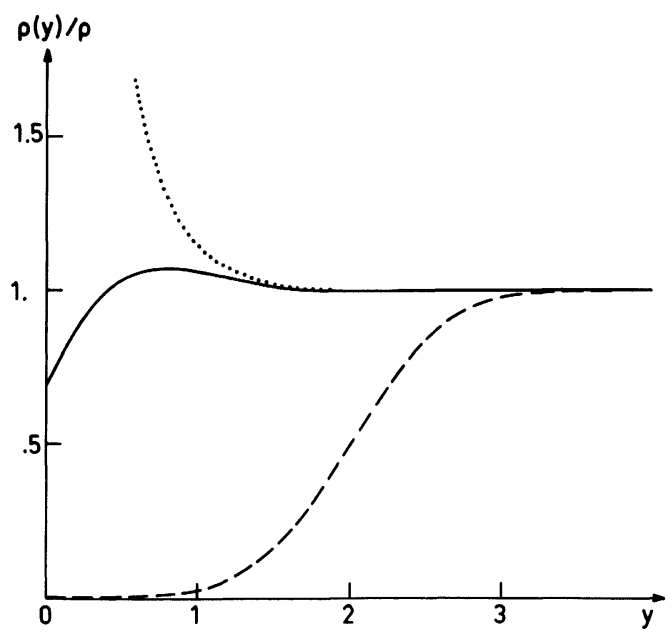

Fig. 1. - The density profile as a function of the distance to the wall. The full line is for a neutral system (Eq. (2.5)), the dashed line for a large defect of particles (Eq. (3.5)), the dotted line for a large excess of particles (Eq. (3.7)). 
$\rho(y)$ goes to its bulk value $\rho$. For arbitrary values of $y$, the integral (2.5) must be performed numerically. The resulting density profile $\rho(y)$ is plotted in figure 1 . It shows an oscillation. Using (2.5), one can check after some manipulations that the total charge near the wall is zero

$$
a \int_{0}^{\infty}[\rho(y)-\rho] d y=0 .
$$

3. Non-neutral system. - We still consider a system of $N$ particles of charge $e$ in a disc of radius $R$. The disc is filled with a uniform background of total charge $-N_{\mathrm{b}} e$, and now $N_{\mathrm{b}}$ may be different from $N$. We define the length $a$ as $a=R / \sqrt{N}_{\mathrm{b}}$ and the background density as $\rho=N_{\mathrm{b}} / \pi R^{2}=1 / \pi a^{2}$. The distance $r$ to the disc centre is expressed by the dimensionless variable $z=r / a$. When $\Gamma=2$, instead of (2.1) one finds for the one-body density $\rho(z)$

$$
\frac{\rho(z)}{\rho}=\mathrm{e}^{-z^{2}} \sum_{n=0}^{N-1} \frac{z^{2 n}}{\gamma\left(n+1, N_{\mathrm{b}}\right)} .
$$

We choose an excess of defect $\left|N-N_{\mathrm{b}}\right|$ of the order of $\sqrt{N}_{\mathrm{b}}$, and define a surface charge density $e \sigma$ by $\sigma=\left(N-N_{\mathrm{b}}\right) / 2 \pi R$. The dimensionless distance to the disc edge is $y=\sqrt{N}_{\mathrm{b}}-z$. We take the thermodynamic limit of (3.1) as $N_{\mathrm{b}} \rightarrow \infty$ for fixed values of $\rho, \sigma$, and $y$. Through a minor modification of the argument of section 2 , we now obtain

$$
\frac{\rho(y)}{\rho}=\frac{2}{\sqrt{\pi}} \int_{-\pi a \sigma \sqrt{2}}^{\infty} \frac{\exp \left[-(x-y \sqrt{2})^{2}\right]}{1+\Phi(x)} \mathrm{d} x .
$$

At the wall on the disc edge, the density becomes

$$
\rho_{\mathrm{w}}=\rho(0)=\rho \ln \frac{2}{1-\Phi(\pi a \sigma \sqrt{2})},
$$

and the corresponding kinetic pressure is given by (1.3). For large values of $y$, i.e. far away from the wall, $\rho(y)$ goes to the background density $\rho$; therefore the net charge density $e[\rho(y)-\rho]$ does concentrate near the walls, as it should. Using (3.2), one can check that the total surface charge density has the right value :

$$
a \int_{0}^{\infty}[\rho(y)-\rho] \mathrm{d} y=\sigma .
$$

The detail of the shape of $\rho(y)$ can be obtained by numerical integration of (3.2), for every value of $\sigma$. Here, we consider explicitly two extreme cases, as follows.

If there is a large defect of particles ( $\sigma$ large and negative), it is convenient to introduce the neutrality radius $R_{0}$ defined (in the thermodynamic limit) by
$R-R_{0}=\pi a^{2}|\sigma|$; this value is such that the disc of radius $R_{0}$ contains a background charge just opposite to the total charge $\mathrm{Ne}$ of the particles. Within the integration range of $x, \Phi(x)$ can be.replaced by 1 , and one finds

$$
\frac{\rho(y)}{\rho}=\frac{1}{2}\left\{1+\Phi\left[\left(y-\frac{R-R_{0}}{a}\right) \sqrt{2}\right]\right\} .
$$

This density $\rho(y)$ is plotted in figure 1 . The particles neutralize the background up to the neighbourhood of the neutrality radius $R_{0}$, where the particle density falls to zero on a distance of the order of $a$. Equation (3.5) can also be obtained by considering from the start a disc of infinite radius which gives a distribution [7]

$$
\frac{\rho(z)}{\rho}=\mathrm{e}^{-z^{2}} \sum_{n=0}^{N-1} \frac{z^{2 n}}{n !}=1-\frac{\gamma\left(N, z^{2}\right)}{(N-1) !} ;
$$

in the neighbourhood of $z=\sqrt{N}$, using (2.3), one recovers (3.5).

If there is a large excess of particles ( $\sigma$ large and positive), $\rho(y)$ approaches the limiting form

$$
\frac{\rho(y)}{\rho}=\frac{2}{\sqrt{\pi}} \int_{-\infty}^{\infty} \frac{\exp \left[-(x-y \sqrt{2})^{2}\right]}{1+\Phi(x)} \mathrm{d} x .
$$

The integral (3.7) has been performed numerically and the resulting density $\rho(y)$ is plotted in figure 1 ; it diverges for small values of $y$. The excess charge is still concentrated near the wall, even in that extreme case.

For a non-neutral system, by a straightforward extension of the scaling argument [5] which gave (1.2), one obtains a thermal pressure

$$
p^{(\theta)}=\left(k_{\mathrm{B}} T-\frac{1}{4} e^{2}\right) \rho+\pi e^{2} \sigma^{2}
$$

The last term in (3.8) has the correct form [8,9] of the electrostatic pressure created by the excess charge $2 \pi R e \sigma$ localized at the surface and having an electrostatic energy - $(1 / 2)(2 \pi R e \sigma)^{2} \ln (R / L)$.

Choquard et al. have derived the identity [4]

$$
p^{(\mathbf{k})}-p^{(\theta)}=2 \pi e^{2} \rho a^{2} \int_{0}^{\infty}[\rho(y)-\rho] y \mathrm{~d} y .
$$

Using (1.3), (3.3), (3.8) and (3.2), after some manipulations in the integral in (3.9), it is possible to check that this identity is indeed satisfied at $\Gamma=2$.

Finally, note that a surface charge density $e \sigma$ in a semi-infinite plasma can be induced by a surface charge density - $e \sigma$ on the wall. Therefore, equation (3.2) also gives the density profile near a charged wall.

Acknowledgments. - Studying a non-neutral system was suggested to the author by J. L. Lebowitz. 


\section{References}

[1] Alastuey, A. and Jancovici, B., J. Physique 42 (1981) 1; JANCOvici, B., Phys. Rev. Lett. 46 (1981) 386.

[2] Menta, M. L., Random Matrices (Academic, New York) 1967, and references quoted there.

[3] Navet, M., Jamin, E. and Feix, M. R., J. Physique Lett. 41 (1980) L-69.

[4] Choquard, Ph., Favre, P. and Gruber, Ch., J. Stat. Phys. 23 (1980) 405 .
[5] Hauge, E. H. and Hemmer, P. C., Physica Norvegica 5 (1971) 209 , and references quoted there.

[6] ERdelyI, A., Higher Transcendental Functions, vol. 2 (McGrawHill, New York) 1953.

[7] Ginibre, J., J. Math. Phys. 6 (1965) 440.

[8] Lebowitz, J. L. and Lieb, E. H., Phys. Rev. Lett. 22 (1969) 631.

[9] LieB, E. H. and Narnhofer, H., J. Stat. Phys. 12 (1975) 291. 